\title{
A telepathology based Virtual Reference and Certification Centre for DNA image cytometry
}

\author{
G. Haroske ${ }^{\mathrm{a}, *}$, Francoise Giroud ${ }^{\mathrm{b}}$, \\ K.D. Kunze ${ }^{\mathrm{c}}$ and W. Meyer ${ }^{\mathrm{c}}$ \\ a Institute of Pathology, Dresden-Friedrichstadt \\ General Hospital, Germany \\ ${ }^{\mathrm{b}}$ Institute Albert Bonniot, Faculté de Médecine, \\ Université Joseph Fourier, Grenoble, France \\ c Institute of Pathology, Dresden University of \\ Technology, Germany
}

An increasing need for flexible consultation between pathologists, including the application of fast evolving supplementary technologies, has been identified during the last years. Although pathology is already one of the most advanced application of telemedicine there is more to come from the fast evolution towards computerized microscope image analysis: A reproducible quantification of measurable descriptors of the lesions in cells and tissues (so-called biological markers) is an indispensable adjunct to routine diagnostic application. Among such quantitative methods DNA image cytometry is increasingly applied by pathologists for assistance in diagnostics.

As for other pathological issues, too, a reference center for the clinical application of DNA image cytometry might be therefore of utmost value for pathologists using that method.

Based on advanced telematic technologies, a Virtual Reference and Certification Center (VRCC) could be installed for certifying the cytometry hardware and software, the analytical procedures, and the basic interpretation of the results. It will be designed to be operated as a non-attended service, based on quantification servers accessible via Internet round the clock. The VRCC will supply appropriate standardization and normalization materials and run a GroupWare platform for consensus making by experts.

Keywords: Telepathology, DNA image cytometry, reference center, certification center

\footnotetext{
*Corresponding author: Gunter Haroske, M.D., Ph.D., Institute of Pathology, Dresden-Friedrichstadt General Hospital, Friedrichstr. 41, D-01067 Dresden, Germany. Tel.: +49 351480 3770; Fax: +49 351480 3799; E-mail: gunter.haroske@khdf.de.
}

\section{Introduction}

In the overall medical strategy, improvement in the quality and reproducibility of pathological examinations is a continuous concern that has taken a significant advantage of the recent multimedia technologies (telepathology, reference image banks, case reports databases). Not only the consultation for second opinion or consensus making, but also the application of quantifiable so-called biomarkers can and will improve quality and reproducibility of diagnostics. As one of such quantitation methods DNA image cytometry is increasingly applied by pathologists for assistance in diagnostics. It helps in grading of malignant tumors, as well as in identifying malignancy in borderline lesions and dysplasias or in therapeutic follow-up.

Possible errors in DNA-measurements that are neither identified nor adequately corrected by the user can result in misinterpretation with disastrous clinical consequences. Clinical pathologists applying DNA cytometry are often not able to check all key elements of their laboratory practice, and the performance of their image analysis workstation as well. For optimizing the quality of DNA analysis standardized methodological tests and assistance in the diagnostic interpretation of the cytometric results are imperative.

On one hand, consensus guidelines already exist on standardization of diagnostic DNA image cytometry [7,13], and further European efforts are on the way for implementation of quality control and quality assurance procedures. On the other hand, the diagnostic interpretation of DNA measurements may be very complex, necessitating both biological and methodological knowledge at a high level of competence [3, 10].

As for other pathological issues, too, a reference center for the clinical application of DNA image cytometry might be of utmost value for pathologists using that method. Due to variability in cytological ma- 
terial handing and technological heterogeneity of cytometry devices, both the standardized quality control procedures and the diagnostic assistance by human experts remained rather problematic so far. The rather recent telepathology technology of Remote Quantitation based on standardized evaluation tools could pave the way for solving those problems [15].

A client-server system EUROQUANT for remote DNA ploidy analysis has been developed [12] that provides the following benefits: (1) DNA measurements can be compared independently of the type of machinery used for data acquisition, (2) this occurs at the most recent know-how, (3) diagnostic conclusions can be drawn on a statistic basis, and (4) the quality control can be brought steadily forward to routine practice of diagnostic DNA image cytometry.

In such a way the intercomparability of results obtained in different laboratories is the basis for the application of standardised diagnostic classification systems and therapeutic schemes. According that concept further quantitation servers are under testing, which allow analysing specific methodological aspects of DNA image cytometry $[8,14]$.

A new concept of a Virtual European Reference and Certification Centre (VRCC) will now be presented with an expanded EUROQUANT like server as a core, operated on behalf of an international scientific society by a group of experienced scientists, and using a reference slide system recommended by the PRESS project $^{1}$ [7]. Some of its key elements have already been developed and tested successfully during the EC funded EUROPATH project ${ }^{2}$.

That reference centre will be, both internally and externally, completely rely on advanced telepathology technologies, enabling each pathologist to get assistance in his daily routine.

\section{The concept of a Virtual Reference and Certification Centre}

As any other reference centre in diagnostic pathology the VRCC, too, should provide a requester with:

- consultation in diagnostic problems,

- consultation in methodology,

- training in methodology,

- documentation of quality control and quality assurance procedures, and

- supply of test material.

\footnotetext{
${ }^{1}$ The PRESS project: CEE/BCR-DGXII No MAT1-CT 930010.

${ }^{2}$ The EUROPATH project: CEE DGXIII No HC-1038.
}

It should also offer a platform for consensus derivation for experts concerned with that special field, and should be able to give advice in certification and accreditation problems for those authorities being responsible for supervising the good laboratory practice. As a special feature the VRCC could also provide the users with certified quantitation software tools.

Therefore, the VRCC aims to improve the accuracy and reproducibility of DNA cytometry by the implementation of structures, tools and procedures which allow end-users to get their DNA measurements on clinical specimens validated and certified, which provide industrial suppliers with normalised QC labels for the clinical use of their instruments and dedicated software, and which offer national and international authorities a certification service for the clinical use of the method mentioned above.

The VRCC is designed to be operated as a nonattended service, based on Quantitation Servers accessible through Internet round the clock. Because it is based on automated devices, operating in a distributed manner and mostly non-attended in a network, that reference centre is really a virtual one. The link between those tools and the interaction of human experts can be assured by telepathology techniques, e.g., by offering multimedia conferencing facilities to the experts concerned [20].

However, such a VRCC needs additional, "classical" structure elements for its operation. It has to be supervised by a scientific framework, assuring the VRCC operation at the most current scientific level. It has furthermore to be run in the real world of networking, i.e., a service provider has to be found for the technological background, whereas an editorial board is responsible for realizing the scientific outlines in the routine work of the VRCC. In Fig. 1 the schematic structure of the VRCC is shown.

\section{The key elements of the VRCC}

\subsection{Multimedia communication platform}

Such a platform consists of web-based mechanisms for rapidly reaching consensus and offering advice about all successive steps of DNA image cytometry, i.e., choosing the appropriate probe and reagents, preparing the biological materials, performing the staining reaction, acquiring images, measuring the relevant parameters, extracting significant data (coefficient of variation - cv, DNA-index, etc.), choosing per- 


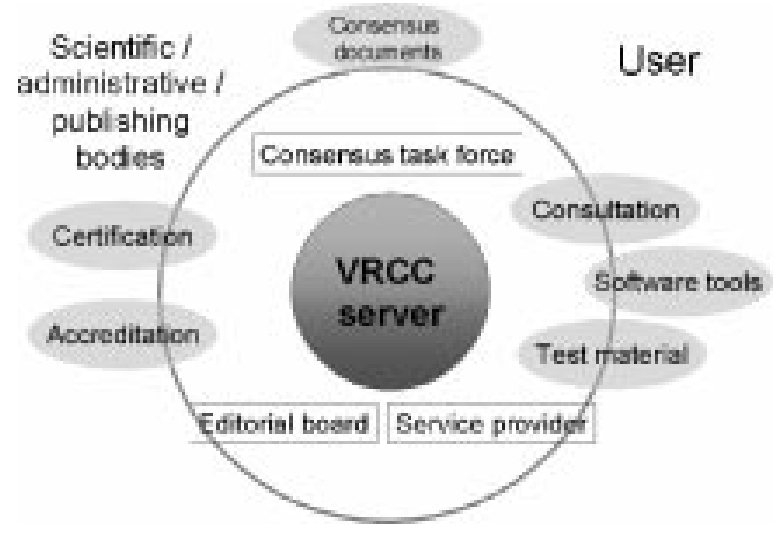

Fig. 1. Schematic structure of a VRCC and its relations to users.

tinent representation of data information (histogram, scattergram, etc.) and deriving the medical conclusions. That platform will be operated by a GroupWare making possible to consistently and synchronously interact in Areal-time through Internet protocols (and next generation browsers).

The Multimedia communication platform is also acting as an Internet portal for any pathologist or scientist interested in DNA cytometry in general. As a first working model for such a platform the PATHCONSULT server (http://pathconsult.imag.fr) gives a first impression how to work with such a communication tool.

\subsection{Quantitation server}

The Quantitation server of the EUROQUANT type (http://euroquant.med.tu-dresden.de) [15] is meant to act as the general methodological tool for performing DNA image cytometric measurements and evaluating them according specified criteria for consistency with Quality Assurance guidelines [7,13]. It will be used both by requesting pathologists, experts in consensus task forces, and by hard- and software suppliers in the field. The VRCC quantitation server may physically be distributed at several different servers deployed across the Internet, as it can recently be seen with EUROQUANT, ATCDI (http://pcsic2/) and QACODIC (http://pcgermong.imag.fr/ qacodic) [8] servers, each providing several specific features of quality control and diagnostic interpretation in DNA image cytometry.

The quantitation server itself consists of a set of several tools for communication between users, and evaluation of DNA measurements, including user-specific databases.

\subsection{Tumor marker database}

That database serves for actually collecting certified DNA measurement data from specific pathologic lesions, together with relevant end-point, grading and staging data in an anonymous manner, as to provide data for derivation of rules diagnosis and prognosis making. The population of such a database is expected to be made by both consensus task forces and individual pathologists interested in scientific evaluation of their clinical material. A pilot example for such a tumor marker database and its combination with a quantitation server can also be seen (and used) in the database consultation section of the PATHCONSULT server mentioned above.

\subsection{Quality control framework}

In order to achieve reliable results of DNA image cytometry, the conditions for reproducible preparation, measurement and interpretation have to be worked out, carefully followed and checked at various steps of the process in certain time intervals. A quality control framework is aimed at assuring the uniformity and reliability of the diagnostic outcome by a continuous application of specific quality control test with feedback mechanisms to the entire process. It is therefore aimed at retrieving the error sources at all stages of the methodological and diagnostic procedure. The framework of such tests, elaborated and tested in the PRESS project, and agreed in a slightly modified version in the ESACP consensus [7], will be used for the VRCC operation, too.

\subsection{Test material laboratories}

A few laboratories, accredited by a scientific body, e.g., ESACP, serve for the manufacturing and deployment of the test material required for running the appropriate quality control protocols.

\subsection{Editorial board}

The editorial board consists of scientists, approved by a scientific society, e.g., ESACP, and responsible for the operation of the VRCC tools in a scientific manner, i.e., according to the recommendations and guidelines, elaborated and confirmed scientifically. It is that board which decides on the configuration of the quality control tests, their acceptance thresholds and the performance of certification and accreditation. It is also responsible for reply to consultation requests from users. The Editorial board will also benefit from operating the Multimedia communication platform. 


\subsection{Service provider}

A service provider is needed for the technological and commercial aspects of running a VRCC, especially for keeping paste with the emerging technologies in telepathology and Internet communication, as well as for invoicing for certification procedures and for protection of property rights. The provider should have an independent, non-profit status, e.g., by having the legal status of a foundation.

\section{Results from testing VRCC elements in a routine environment}

\subsection{Remote consultation}

Besides the telediagnosis on intraoperative frozen sections, the teleconsultation is gaining considerable momentum in telepathology applications, often with an attempt towards quality control [5]. Several projects are in operation, both experimental $[9,26,28]$ and full service providing ones. Most of them are based on static telepathology procedures, the more general services are using Internet protocols for assuring a high degree of interoperability [17,23,30,33].

The PATHCONSULT server offers REMCON for remote consultation procedures based on Internet and multimedia mailboxes. That facility was successfully tested by groups of peers and consultants for specific groups of tumors [28].

\subsection{Remote data analysis}

The DNA image cytometry measurements are evaluated automatically without human interaction. Two different options are offered to have diagnostic specimens analyzed:

(1) By sending microscopic images, grabbed from specimens specifically stained, to the server for image analysis, objects measurements, and analysis of the performance of measurements.

(2) By sending measurement data (integrated optical density, nuclear area, and coding of each nucleus measured) obtained by any DNA cytometry device to the server for data analysis and quality control in a standardized procedure.
There is no specific hard- or software needed by the user in addition to a telepathology/cytometry workstation equipped with an Internet browser. For more privacy the user can also have ISDN access with the same Internet technology or Internet SSL encryption procedures.

The principal sequence in using the server is always the same: (1) transfer of images or data to the server, (2) import into the server's database, (3) ploidy analysis, check of performance standards as well as quality control, and (4) downloading the results. That full sequence is illustrated in Fig. 2.

After the images have been transferred to the server's mailboxes, the user can operate the server by his client computer for remote quantitation. The images are automatically segmented, and the segmentation masks are displayed to the user together with a table of options for selecting reference cells, or for deleting objects from analysis. Any serious errors in the gain and offset control of the imaging device at the client workstation are already indicated in the processed image at the server and announced to the user.

The analysis of the data gathered from images at the server or transferred from the client cytometry workstation is started by an import of the key data (nuclear area, integrated optical density) from each cell into the server's databases. The functions of the server yield to a comprehensive selection of primary figures (numerical data and graphs), backed up by a wide range of quality assurance data, according to generally accepted guidelines for good measurement practice. In Fig. 3, a result display page is shownpartly. All values exceeding guideline thresholds (recommended by the ESACP [7,13]) are flagged; help is offered for finding the reason of those deviations, e.g., an undercorrected glare effect in the microscope.

The results remain stored in a multimedia mailbox of the server, from where they can be downloaded by the user at any time.

Until now, more than 40 laboratories from Europe, USA, and Asia, working with 13 different types of cytometry systems, have been registered and authorized for access at the quantitation server. They performed analyses on about 4300 ploidy data sets successfully. For five out of them, the server has been incorporated in the quality control of daily routine in DNA image cytometry. The server is online free-of-charge 24 hours the day.

In Table 1 the variety of image cytometry workstations operated by registered EUROQUANT users is given. 
Telepathology Workstation

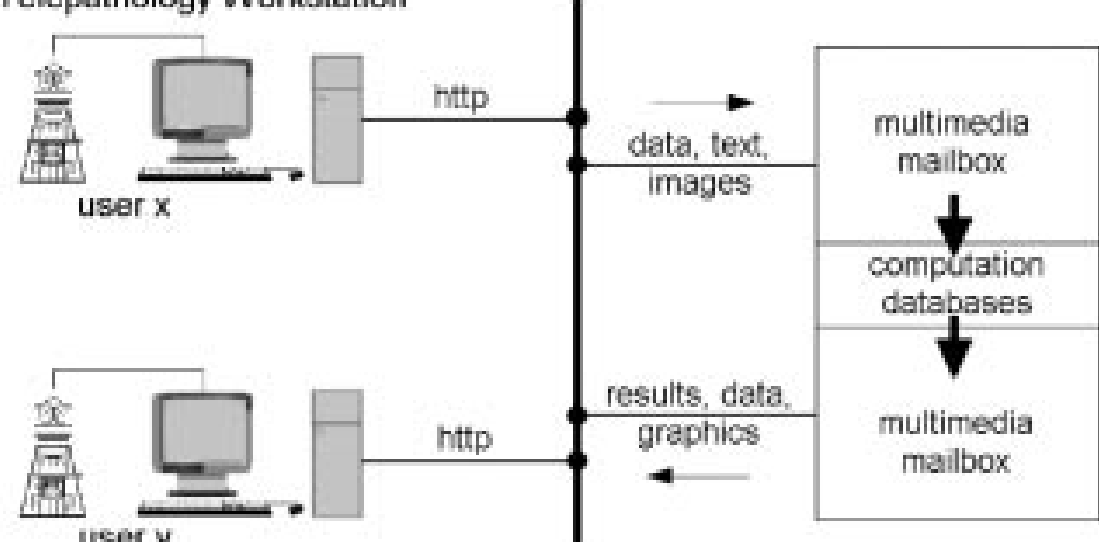

user y

Cytometry Workstation

Internet / ISDN

EUROQUANT

Fig. 2. The quantitation server can be accessed by different types of users via the Internet.

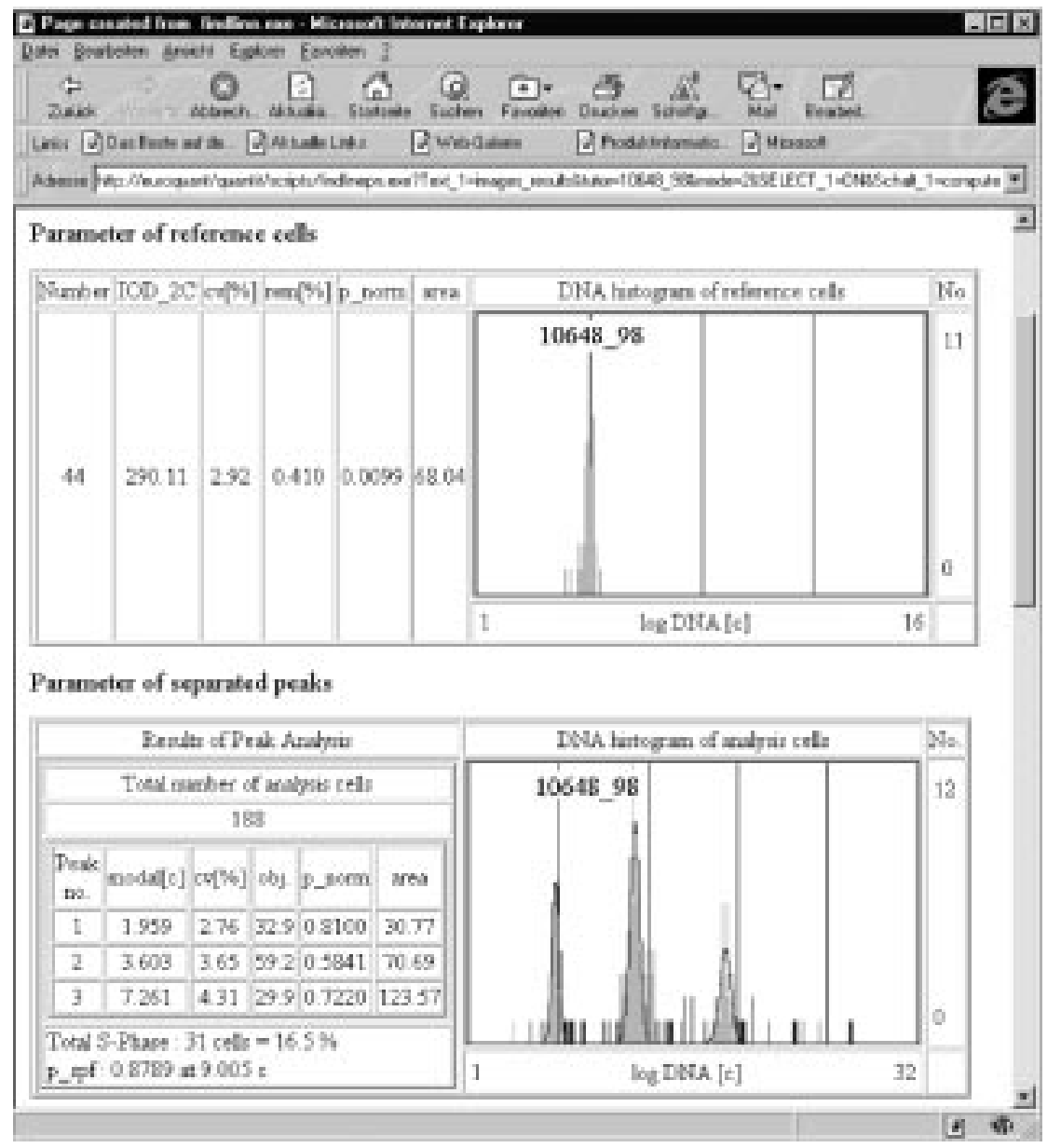

Fig. 3. A web page with results of a EUROQUANT DNA cytometric analysis. 
Table 1

Image cytometry systems operated by EUROQUANT users

\begin{tabular}{llc}
\hline Type of system & Delivered by & $\begin{array}{c}\text { Number } \\
\text { of users }\end{array}$ \\
\hline AxioHOME & Zeiss/Alcatel (Germany/France) & 3 \\
ACAS & Ahrens (Germany) & 1 \\
CAS 160 & Becton \& Dickinson (USA) & 3 \\
CAS 200 & Becton \& Dickinson (USA) & 2 \\
CIRES/QUIC-DNA & Zeiss/Roche (Germany/France) & 3 \\
CM-1 & Hundt (Germany) & 3 \\
KONTRON & Zeiss (Germany) & 1 \\
OPTIMAS & Optimas (USA) & 5 \\
Pathology & Roche (France) & 1 \\
Workstation & & \\
Quanticyt & UDDL (n.a.) & 1 \\
Qploidy & Leica (Germany) & 1 \\
SAMBA & Samba Technologies (France) & 4 \\
VITUM & SIS (Germany) & 1 \\
\hline
\end{tabular}

Table 2

Survey of the routine material investigated by different users

\begin{tabular}{lc}
\hline Organ/pathology & Number \\
\hline Ascitic fluid & 25 \\
Broncho-alveolar-lavage & 2 \\
Cervical smears & 238 \\
Cervical scrapings, disintegrated & 100 \\
Mammary carcinoma FNAB/imprint & 1550 \\
Pleural effusions & 106 \\
Prostatic cancer FNAB & 5 \\
Sweat gland tumours, disintegrated & 86 \\
Thyroid nodules FNAB & 8 \\
Urinary bladder washings & 163 \\
Oral cavity tumours & 303 \\
\hline
\end{tabular}

On an average, a dialogue between the user and the quantitation server for transferring and importing measurement data of one specimen, as well as for calculation of a DNA histogram takes less than two minutes. The calculation of histogram data and their display require less than 10 seconds. Image transfers require more time, dependent on the type of transfer medium and the image size.

DNA data and images from a wide variety of diagnostic material were analyzed. The types of material are shown in Table 2.

\subsection{Quality control framework, tools, and diagnostic advice}

A user may have quality control tools at different levels of the cytometric and diagnostic process. Those

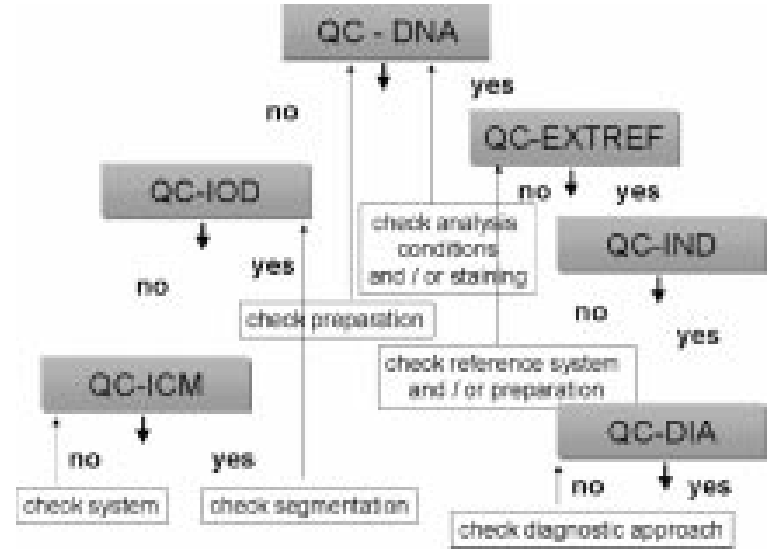

Fig. 4. Framework of quality control tests.

tools are meant to act in a framework, demonstrated in Fig. 4.

All those tools share the common evaluation of primary DNA measurements by the server. (1) Technological settings of the client cytometry workstation can be tested by the remote image quantitation tools, with genuine PRESS type test slides (for QC-ICM, QC-IOD at the QACODIC server, for QC-DNA at the QACODIC and EUROQUANT server, too). The user can check the stability of the preparation, fixation, staining, and further technological aspects of the cytometry process by means of calibration material and check sample specimens, recommended by the European Society of Analytical Cellular Pathology [7]. (2) The quality of the measurement of each single specimen is checked steadily during the normal analysis (see below). (3) The quality of the rescaling process of the DNA axis is controlled by means of several Levy-Jennings charts, built up from results of the users own measurements hold at the databases of the server. (4) Finally, a series of images from diagnostic specimens is hold in the multimedia mailboxes for testing the diagnostic vigilance of the users.

Having analyzed a certain amount of specimens with non-pathological conditions among them, the user can be supplied by the server with data used for rescaling of the primary measurement data into units of "c" (DNA content). By such a procedure the DNA data become intercomparable with those from other users obtained on quite different cytometry devices, making such data to highly standardized ones.

The VRCC servers works completely independent of any commercially offered cytometry device. Their functionality is based on the latest scientific knowledge and consensus agreement in the field. Therefore, the 
Table 3

Quality control variables of the QC-DNA test for different users

\begin{tabular}{cccccccc}
\hline user code & cvref $_{\text {intra }}$ & cvref $_{\text {inter }}$ & cv2 $_{\text {intra }}$ & cv2 $_{\text {inter }}$ & ${\text { cv } 4 c_{\text {intra }}}$ & cv4c $_{\text {inter }}$ & cvr42 $_{\text {inter }}$ \\
\hline $1^{*}$ & 2,125 & $\mathbf{3 , 1 6 6}$ & 2,172 & $\mathbf{2 , 2 3 3}$ & 1,844 & $\mathbf{2 , 1 4 6}$ & 0,462 \\
$1^{* *}$ & 1,263 & 2,242 & 1,565 & 0,722 & 1,359 & 0,745 & 0,175 \\
3 & 3,376 & $\mathbf{6 , 4 2}$ & 4,256 & $\mathbf{4 , 1 8}$ & 2,51 & $\mathbf{4 , 2 0 7}$ & $\mathbf{1 , 7 5 4}$ \\
4 & 4,699 & $\mathbf{4 , 6 5 9}$ & 4,535 & $\mathbf{3 , 9 1}$ & 4,004 & $\mathbf{4 , 6 6}$ & $\mathbf{1 , 2 4 4}$ \\
5 & 2,472 & $\mathbf{5 , 5 9 5}$ & 2,661 & $\mathbf{2 , 0 5 7}$ & 2,029 & $\mathbf{2 , 4 3}$ & 0,399 \\
10 & 1,648 & 1,93 & 1,904 & 0,698 & 1,958 & 0,697 & 0,581 \\
12 & 1,932 & $\mathbf{3 , 2 8 7}$ & 1,831 & 0,53 & 1,948 & 0,376 & 0,479 \\
$13^{*}$ & $\mathbf{7 , 1 7}$ & $\mathbf{6 , 2 5 5}$ & 4,145 & $\mathbf{8 , 4 4 1}$ & 4,895 & $\mathbf{8 , 5 9 9}$ & $\mathbf{2 , 0 4 1}$ \\
$13^{* *}$ & 3,692 & $\mathbf{4 , 8 9 8}$ & 2,236 & $\mathbf{2 , 1 3 3}$ & 2,455 & $\mathbf{2 , 4 6 7}$ & 0,857 \\
\hline
\end{tabular}

cvref $_{\text {intra }}$, intra-imprint cv of reference cells, cvref $_{\text {inter }}$, inter-imprint cv of reference cells; cv2 $\mathrm{c}_{\text {intra }}$, intra-imprint cv of diploid hepatocytes; $\mathrm{cv} 2 \mathrm{c}_{\text {inter }}$, inter-imprint cv of diploid; $\mathrm{cv} 4 \mathrm{c}_{\text {intra }}$, intra-imprint $\mathrm{cv}$ of tetraploid hepatocytes; $\mathrm{cv} 4 \mathrm{c}_{\text {inter }}$, inter-imprint $\mathrm{cv}$ of tetraploid hepatocytes; $\mathrm{cv} 42_{\text {inter }}$, interimprint $\mathrm{cv}$ of the ratio between tetraploid and diplod hepatocytes;

Values above the recommended tresholds are printed in bold.

*First run; ${ }^{* *}$ second run.

VRCC servers are an objective tool for an international methodological standard.

Several rounds of Quality Control Programs, run according the ESACP recommendations [7] by some of the users, demonstrated a sufficient level of precision and accuracy in the measurements of the majority of the users. In those users who failed to meet the guideline threshold, a second run showed that a feedback occurs while spotting systematic errors, leading to successfully passing the repeated tests.

In Table 3 the appropriate figures are given for the general test of DNA preparation and measurement (QC-DNA). None of the users had to proceed to the more machine-oriented test of QC-IOD or QC-ICM.

The permanent quality control of the daily routine measurement is provided by several server tools. On average, the quality-related variables in routine cases do meet the ESACP consensus guidelines, too (Table 4).

A comprehensive graphical display allows the user to make cross checks of possible error sources in his measurements, e.g., by comparing the characteristics of reference cells with the peaks of analysis cells, by checking glare effects versus variability due to methodological procedure compared to a possible biological variability (Fig. 5). The basic rationale behind that approach is that a measured variability (e.g., expressed as coefficient of variation) of a peak in the DNA histogram is the resultant of biological sources and methodological errors. The latter are made visible by the servers analysis functions and display capabilities. In Fig. 5 those influences become obvious in two distinct measurements in breast cancer im-
Table 4

QC variables of individual measurements from 17 users

\begin{tabular}{lccc}
\hline Variable & minimum & mean & maximum \\
\hline number of reference cells & $\mathbf{1 0}$ & 24 & 101 \\
$c v$ of reference cells & 1.31 & 3.69 & $\mathbf{6 . 6 6}$ \\
rem of reference cells & 0.19 & 0.73 & $\mathbf{2 . 2 1}$ \\
corr_coeff area vs IOD & 0.01 & 0.28 & $\mathbf{0 . 7 7}$ \\
p_homogeneity & $\mathbf{0 . 0 0 4}$ & 0.058 & 0.45 \\
number of analysis cells* & 125 & 257 & 312 \\
\hline
\end{tabular}

$\mathrm{cv}$, coefficient of variation [\%]; rem, relative error of the mean [\%]; corr_coeff, correlation coefficient $r, p \_$homogeneity; error probability $p$ that a sequence of measurements is randomly distributed (see Fig. 5).

No recommendation.

prints, showing both considerably high peak cv's. In both measurements glare/diffraction effects, beyond the ESACP guidelines, are responsible for the high peak cv's (Fig. 5b). In the sample 44 an inhomogeneous sampling has an additional share in the $\mathrm{cv}$ of the peak. This is indicated by the statistically significant departure from homogeneity, shown by horizontal bars beneath the graph of the sequential DNA-values (Fig. 5c). The DNA-values at the right are systematically higher than those of the left.

\subsection{Training and self-check facilities}

From the editorial board a case collection will be gathered, consisting of series of images of Feulgen stained specimens from clinical cases with specific diagnostic problems. A user who is willing to check his ability and experience in performing a clinical DNA image cytometry investigation can select the appropri- 

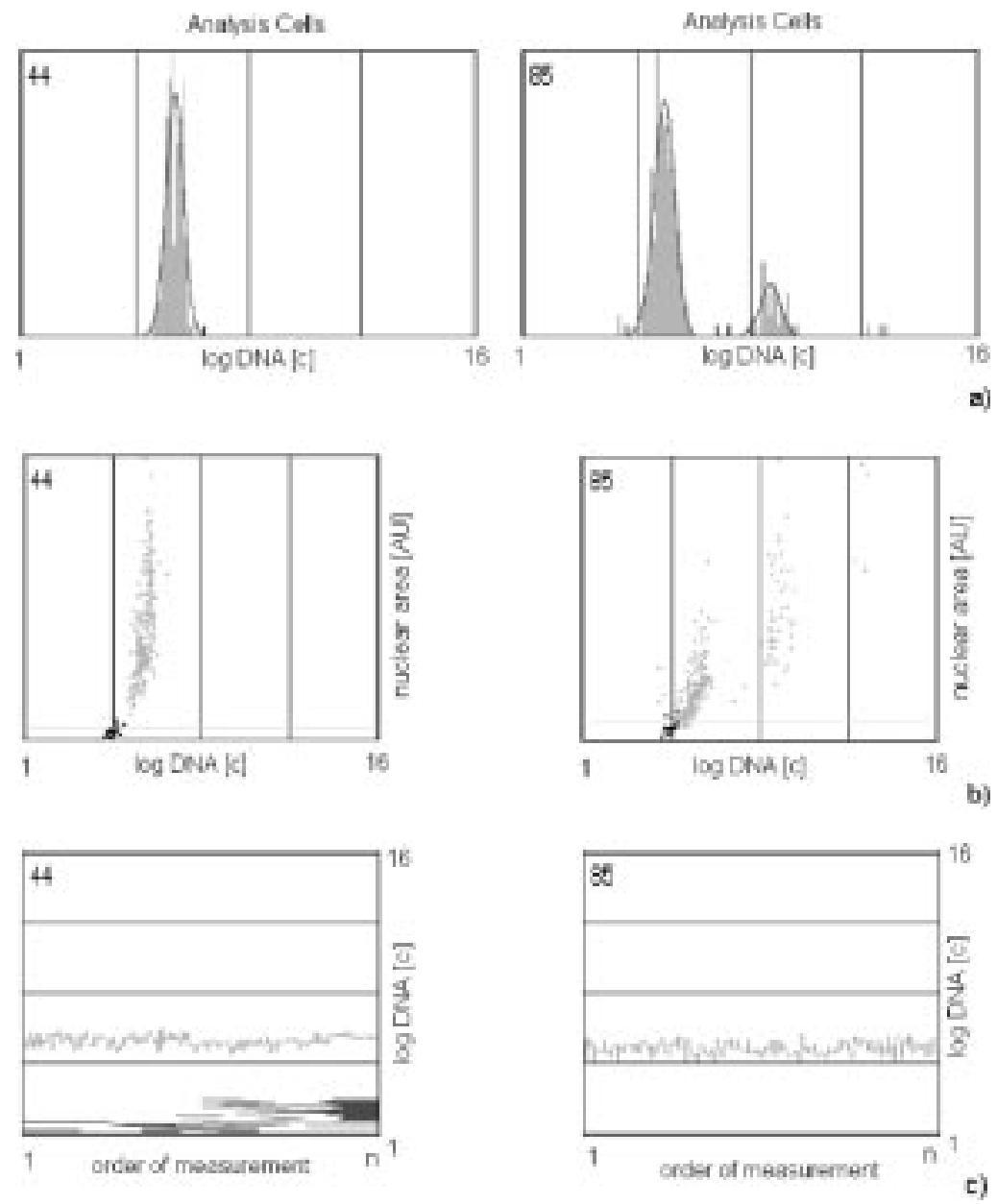

Fig. 5. Part of the EUROQUANT result display, dealing with internal quality control issues. (a) DNA histograms of two samples from breast cancer imprints. Case 44: stemline at $2.55 \mathrm{c}$ with $\mathrm{cv}$ of $5.34 \%$; case 85 : stemline at $2.38 \mathrm{c}$ with $\mathrm{cv}$ of $6.22 \%$; (b) Scattergrams of nuclear area vs. DNA content in those two samples. The deviation of the ellipsoid from the vertical axis, expressed as correlation coefficient $r$, indicates glare and/or diffraction effects beyond the ESACP guidelines in both specimens ( $r=0.56$ in $44 ; r=0.67$ in 85 ). Those effects lead also to a shift in the modal peak value of analysis cells to the right (compared with the smaller reference cells, printed as black dots); (c) Sequential displays of the DNA content of cells within a histogram peak (same measurements as in (a), showing a statistically significant departure from homogeneity in sample 44 ( $p=0.000$ in $44 ; p=0.26$ in 85$)$.

ate images and operate the server for making DNA cytometric measurements. The diagnostic problem is given together with the image series, and the user can decide what cells have to be measured. After having stopped the analysis he will be confronted with the diagnostic consensus of the editorial board, with his own diagnosis, and with the diagnoses of all other users having analyzed the same case so far. In Fig. 6 an image gallery of one of those cases is shown.

\subsection{Data privacy and response time}

An invaluable precondition for a practical application of such quantitation servers, available internation- ally, is the absolute preservation of the user's privacy. Research oriented data analysis in the server databases is accessible for the users only. Their privacy is protected by an authorization process with personal passwords, and by a firewall in front of the server, preventing unwanted access to the users mailboxes. A still higher degree of data protection, also during the data transfer, can be obtained by using ISDN access or of the Internet with SSL procedures [2] or other publickey cryptography [29]. The use of ISDN increases the communication costs, but does not have any influence on the hard- and software configuration. 


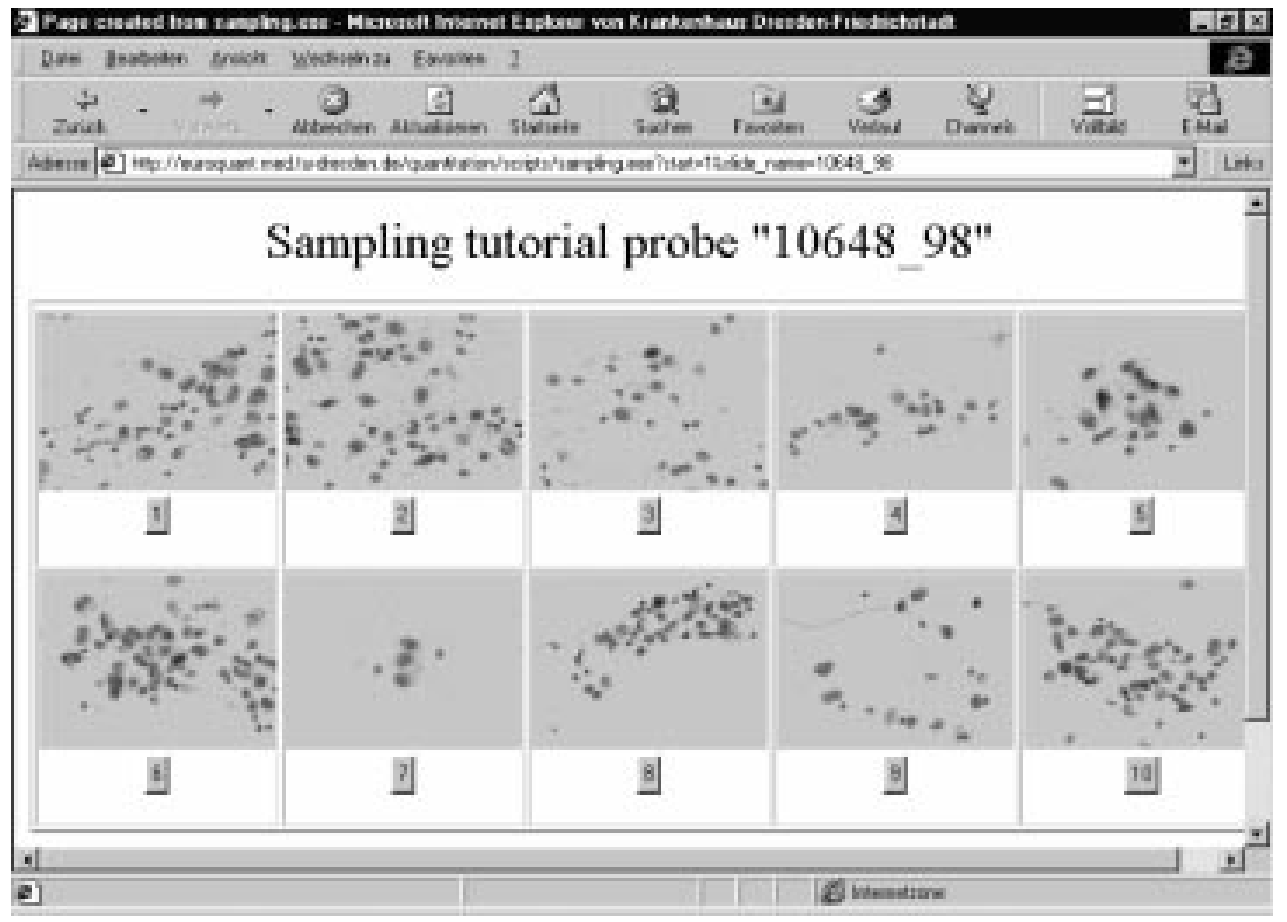

Fig. 6. Image gallery of scenes from a Feulgen stained tumor imprint. The user may select scenes and individual cells for performing a server based DNA cytometry.

\subsection{Feeding marker databases with certified DNA cytometry data}

A specific EUROQUANT functionality is the electronic signature of rescaled measurement data. By that signaturing the data are certified to fulfil quality guidelines as agreed, and are protected against possible manipulation. Only data having that signature are allowed to populate the PATHCONSULT marker data base, as a part of the Case Report facility for scientific studies or publication. More than 20 case reports with DNA cytometry data have been gathered and reviewed by an Editorial Board so far.

\section{Discussion}

In recent clinical pathology the application of adjuvant methods for diagnostics, prognosis prediction and therapeutic follow up is rapidly growing. Due to the frequently high complexity of those methods the single pathologist cannot be familiar with all of them at the same degree. Not only indication may be critical but also the methodological skills and the diagnostic interpretation requires often external advice by specialists from centers of competence. However, most of the rou- tine pathologists will ask rather sporadically for advice in a certain method or a diagnostic problem. A close collaboration between routine pathologists and special centers is therefore rather exceptional.

With the upcoming telepathology the current paradigms could change [18-20].

Among the application fields of telepathology the Remote Quantitation is a comparatively recent one [11, 20]. Because today telepathology is almost exclusively based on the exchange of digitized images [6,18-20, $25,32]$, the parallel use of cytometric or similar techniques together with image transfer seems to be a logical consequence for solving special problems in clinical pathology [24,27]. However the quantitation of such digitized images is faced with several difficulties, most importantly with the lacking interoperability and methodological comparability of a rather broad spectrum of existing cytometry and morphometry devices. Therefore, a client-server concept was designed as to overcome those obstacles [15]. That concept is based on the technological state of the art for existing cytometry workstations spread over university institutes, hospital departments, and private institutes for pathology throughout the world, as well as on the needs of clinical pathologists to get high level assistance in the application of special adjuvant methods for diagnosis 
making, and on the existing achievements in standardization of such methods. A quantitation server does not only allow a remote quantitation of DNA data, but it also facilitates those analyses without any human interaction, i.e., in an objective way. By means of those analyses the pathologist is provided with qualitycontrolled proposals for classifying or typing a certain DNA-histogram. The final diagnostic decision, however, is exclusively in the hands of the pathologist.

A further important aspect for practice is the lack of any time constraints in contacting such a non-attended service, compared with traditional scenarios of Remote Consultation with an expert available in time $[17,23$, 30,33].

Furthermore, a server is able to act as an interface for contributors of data to centralized tumor marker databases, and for an editorial board evaluating the entries to this marker database.

As such a "neutral" platform a quantitation server may enable a very practical application of the concepts in quality control, quality assurance, and good laboratory practice in pathology, especially in diagnostic DNA cytometry [12].

It seems to be a logical consequence to build up a reference center around such a server (or a cluster of servers), combining the Remote Quantitation functions with other advanced telepathology techniques, such as multimedia mailboxes, groupware for consensus making, tumor marker databases, and the Internet portal concept. All the advantages mentioned above for the server itself hold also true for a Virtual Reference and Certification Center. It is likely that this concept can be usefully extended to other fields involving quantitation such as immunocytochemistry and molecular medicine.

On the other hand, with the upcoming turn in telepathology technology towards server-based multipurpose workstations $[1,16,19,20,34,35]$, operated by Internet agents, the VRCC concept fits very well in the general approach of providing collaboration for distant medical professionals [4]. For the first time in the history of diagnostic probes and markers, a dedicated tool and built-in procedures will make it possible to cooperatively establish a "validation electronic dossier" through Internet protocols. In about five years from now, such a dossier may likely become a pre-requisite for medical decision makers to decide whether and why to pay for the probes, reagents and pieces of cytometry equipment in the context of evidence-based medical practice.

\section{References}

[1] R.D. Aller, The pathologist's workstation, Clin. Lab. Med. 17(2) (1997), 201-228.

[2] U.J. Balis, Telemedicine and telepathology, Clin. Lab. Med. 17 (1997), 245-261.

[3] A. Böcking, DNA measurements: when and why?, in: Compendium on Quality Assurance, Proficiency Testing and Workload Limitations in Clinical Cytology, G.L. Wied, C.M. Keebler, D.L. Rosenthal, U. Schenck, T.M. Somrak and G.P. Vooijs, eds, Tutorials of Cytology, Chicago, 1995, pp. 170-188.

[4] V. Della Mea, V. Roberto, A. Conti, L. di Gaspero and C.A. Beltrami, Internet agents for telemedicine services, Med. Inform. Internet Med. 24(3) (1999), 181-188.

[5] P.A. Dervan and R. Wootton, Diagnostic telepathology, Histopathology 32 (1998), 195-198.

[6] T.J. Eide and I. Nordrum, Current status of telepathology. Review article, APMIS 102 (1997), 881-890.

[7] F. Giroud, G. Haroske, A. Reith and A. Böcking, 1997 ESACP consensus report on diagnostic DNA image cytometry. Part II: Recommendations for quality assurance, Anal. Cell. Pathol. 17 (1998), 201-208.

[8] F. Giroud and G. Haroske, Protocoles pour le contrôle et l'assurance qualité pour la cytométrie en image de l'ADNPloïdie. 6th Ann Congress AFC, Dijon, 12-15 Oct. 1999, Anal. Cell. Pathol. 18 (1999), 169.

[9] B.E. Halliday, A.K. Bhattacharyya, A.R. Graham, J.R. Davis, S.A. Leavitt, R.B. Nagle, W.J. McLaughlin, R.A. Rivas, R. Martinez, E.A. Krupinski and R.S. Weinstein, Diagnostic accuracy of an international static-imaging telepathology consultation service, Hum. Pathol. 28(1) (1997), 17-21.

[10] G. Haroske, V. Dimmer, W. Meyer and K.D. Kunze, DNA histogram interpretation based on statistical approaches, Anal. Cell. Pathol. 15 (1997), 157-175.

[11] G. Haroske, W. Meyer and K.D. Kunze, Remote quantitation in DNA image cytometry (Abstract), Anal. Cell. Pathol. 15 (1997), 71.

[12] G. Haroske, W. Meyer, F. Theissig, K. Schubert and K.D. Kunze, Remote quantitation server for quality assurance in DNA ploidy analysis, Analyt. Quant. Cytol. Histol. 20 (1998), 302-312.

[13] G. Haroske, F. Giroud, A. Reith and A. Böcking, 1997 ESACP consensusreport on diagnostic DNA image cytometry. Part I: Basic considerations and recommendations for preparation, measurement and interpretation, Anal. Cell. Pathol. 17 (1998), 189-200.

[14] G. Haroske, Standardisation, quality assurance, and diagnostic interpretation: DNA ploidy as a powerful tumour marker in clinical pathology. 6th Ann. Congress AFC, Dijon, 12-15 Oct. 1999, Anal. Cell. Pathol. 18 (1999), 169.

[15] G. Haroske, W. Meyer, M. Oberholzer, A. Böcking and K.D. Kunze, Competence on demand in DNA image cytometry, Path. Res. Pract. 196 (2000), 285-291.

[16] P. Hufnagel, T.N. Nguyen-Dobinsky and M. Dietel, "Pathologie Arbeitsplatz 2000" für Telemedizin (Abstract), Verh. Dt. Ges. Path. 81 (1997), 661. 
[17] P. Hufnagel, T.N. Nguyen-Dobinsky, W. Striebel and M. Dietel, How the UICC Telepathology Consultation Center (TPCC) works, Path. Res. Pract. 196 (2000), 458.

[18] K. Kayser and C. Kayser, Telepathology - aspects of social influence and quality control, Elec. J. Pathol. Histol. 3(3) (1996), No. 963-04.

[19] K. Kayser and G. Kayser, Basic Aspects of and Recent development of telepathology in Europe with specific emphasis on quality assurance, J. Anal. Quant. Cytol. Histol. 21 (1999), 319-328.

[20] K. Kayser, J. Szymas and R. Weinstein, Telepathology: Telecommunication, Electronic Education and Publication in Pathology, Springer, Heidelberg, New York, 1999.

[21] K. Kayser, G. Kayser and S. Zink, New technical aspects in telepathology, Elec. J. Pathol. Histol. 6(3) (2000), No. 003-04.

[22] A.M. Marchevsky, H. Truong and T. Tolmachoff, A rule-based expert system for the automatic classification of DNA "ploidy" histograms measured by the CAS 200 image analysis system. Cytometry, Comm. Clin. Cytom. 30(1) (1997), 39-46.

[23] F.G. Mullick, P. Fontelo and C. Pemble, Telemedicine and telepathology at the Armed Forces Institute of Pathology: history and current mission, Telemed. J. 2 (1996), 187-193.

[24] M. Oberholzer, H.R. Fischer, H. Christen, S. Gerber, M. Bruehlmann, M. Mihatsch, M. Famos, C. Winkler, P. Fehr, L. Baechtold and K. Kayser, Telepathology with ISDN - A new tool for image transfer in surgical pathology, Hum. Pathol. 24 (1993), 1078-1085.

[25] M.J. Obrien, M. Takahashi, G. Brugal, H. Christen, T. Gahm, R.M. Goodell, P. Karakitsos, E.A. Knesel, Jr., T. Kobler, K.A. Kyrkou, S. Labbe, E.L. Long, L.J. Mango, E. McGoogan, M. Oberholzer, A. Reith and C. Winkler, Digital imagery/telecytology. International Academy of Cytology Task Force summary. Diagnostic Cytology Towards the 21st Century: An International Expert Conference and Tutorial, Acta Cytol. 42(1) (1998), 148-164.
[26] D.H. Okada, S.W. Binder, C.L. Felten, J.S. Strauss and A.M. Marchevsky, "Virtual microscopy" and the internet as telepathology consultation tools: diagnostic accuracy in evaluating melanocytic skin lesions, Am. J. Dermatopathol. 21(6) (1999), 525-531.

[27] K.L. Phillips, L. Anderson, T. Gahm, L.B. Needham, M.L. Goldman, B.E. Wraya and T.F. Macri, Quantitative DNA analysis: a comparison of conventional DNA ploidy analysis and teleploidy, Arch. Anat. Cytol. Pathol. 43 (1995), 288-295.

[28] R.P. Singson, S. Natarajan, J.K. Greenson and A.M. Marchevsky, Virtual microscopy and the Internet as telepathology consultation tools. A study of gastrointestinal biopsy specimens, Am. J. Clin. Pathol. 111(6) (1999), 792-795.

[29] J.S. Strauss, C.L. Felten, D.H. Okada and A.M. Marchevsky, Virtual microscopy and public-key cryptography for Internet telepathology, J. Telemed. Telecare 5(2) (1999), 105-110.

[30] H.A. Swett, L. Holaday, D. Leffell, R.C. Merrell, J.S. Morrow, J.C. Rosser and J. Warshaw, Telemedicine: delivering medical expertise across the state and around the world, Conn. Med. 59(10) (1995), 593-602.

[31] J.H. Tucker, C. Busch, A. Spatz, C. Wells and G. Brugal, An experimental inter-expert telepathology network using static imaging, J. Clin. Path. (2000), submitted.

[32] R.S. Weinstein, A.K. Bhattacharyya, A.R. Graham and J.R. Davis, Telepathology: a ten-year progress report, Hum. Pathol. 28(1) (1997), 1-7.

[33] B.H. Williams, F.G. Mullick, R.L. Becker, R.T. Kyte and A. Noe, A national treasure goes online: the Armed Forces Institute of Pathology, MD Comput. 15(4) (1998), 260-265.

[34] G. Wolf, D. Petersen, M. Dietel and I. Petersen, Telemicroscopy via the Internet, Nature 391 (1998), 613-614.

[35] G. Wolf, I. Petersen and M. Dietel, Microscope remote control with an Internet browser, Anal. Quant. Cytol. Histol. 20(2) (1998), 127-132. 


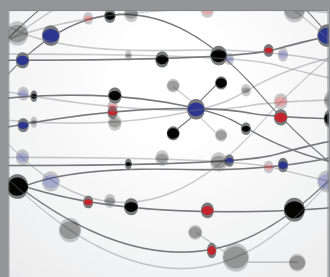

The Scientific World Journal
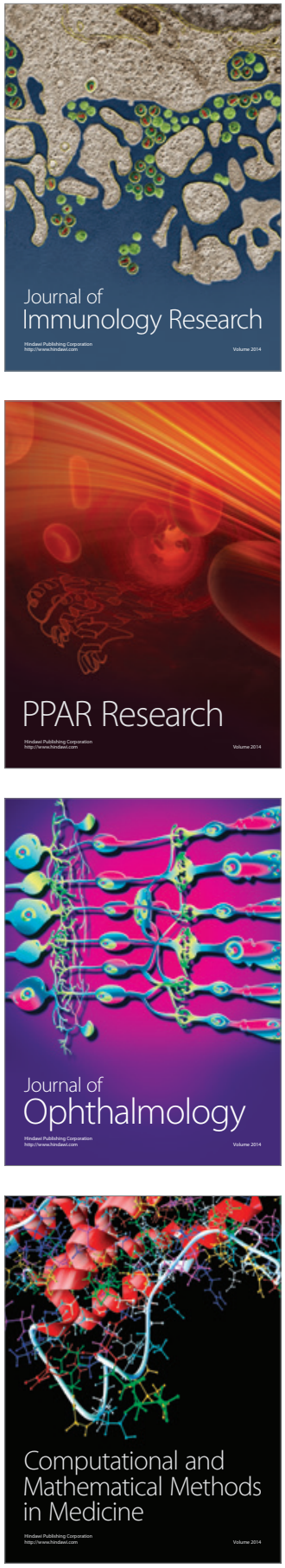

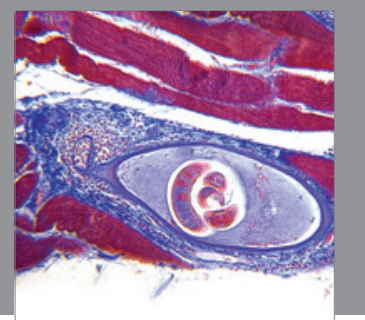

Gastroenterology

Research and Practice
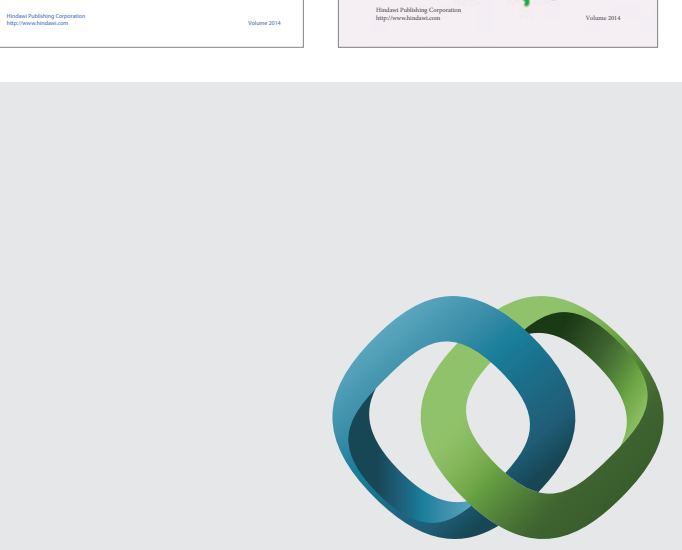

\section{Hindawi}

Submit your manuscripts at

http://www.hindawi.com
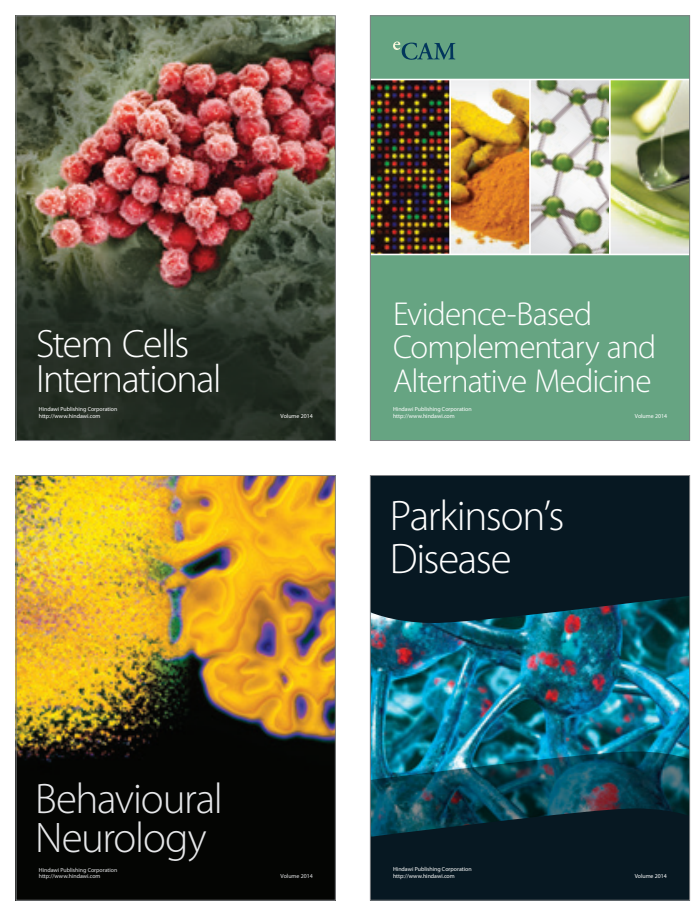

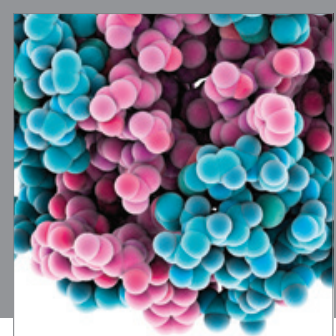

Journal of
Diabetes Research

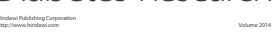

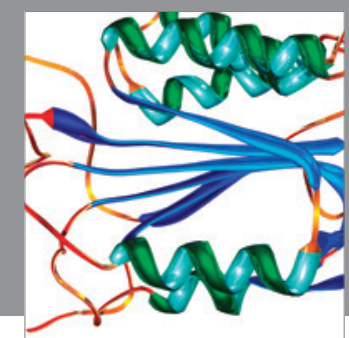

Disease Markers
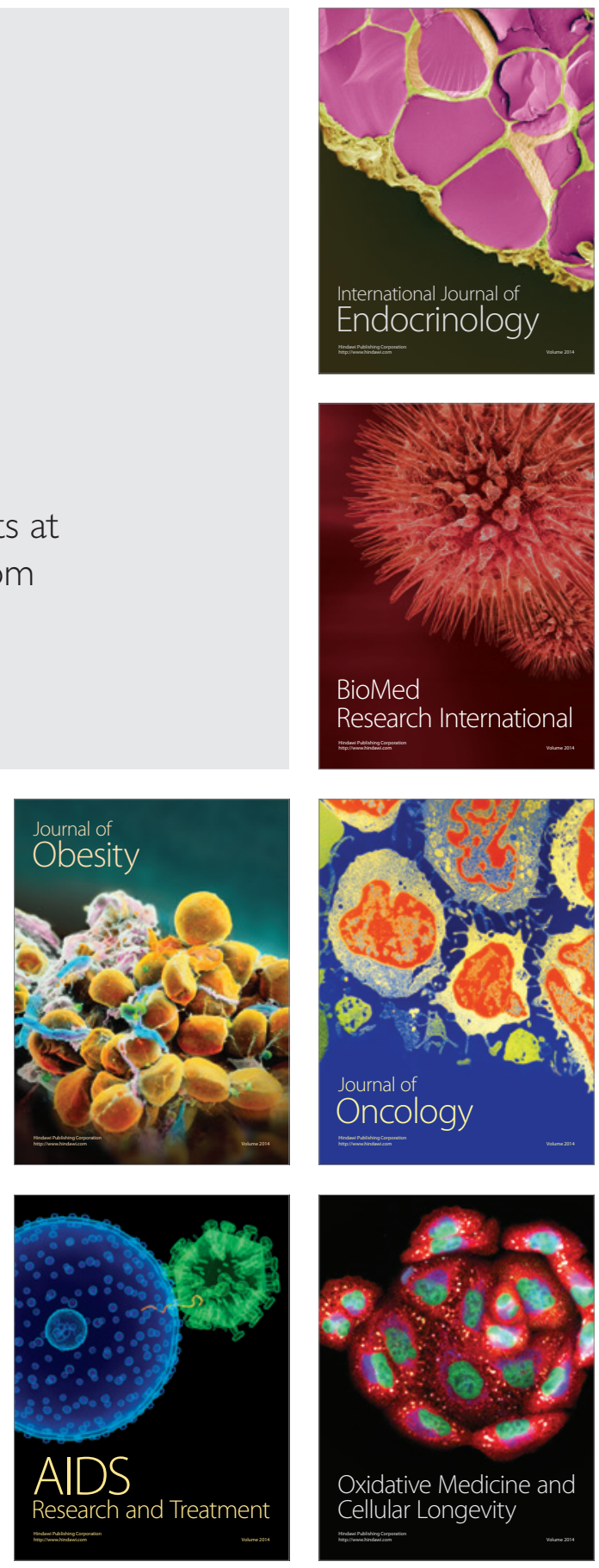